4 Leucht S, Fennema H, Engel RR, Kaspers-Janssen M, Lepping P, Szegedi A What does the MADRS mean? Equipercentile linking with the CGI using a company database of mirtazapine studies. J Affect Disord 2017; 210: 287-93.

Susan Paketci, University of Bristol, Bristol, UK. Email: sp15714@bristol.ac.uk

doi:10.1192/bjp.2021.162

\section{Esketamine: uncertain safety and efficacy data in depression}

11 June 2021

\section{Esketamine efficacy}

Six 4 week efficacy trials have now been published, of which only one reports a statistically significant difference between placebo nasal spray (and antidepressant) and esketamine (and antidepressant) on depression score at 4 weeks. There is debate about whether the 4.0 point difference found constitutes a clinically significant effect, ${ }^{1}$ especially considering the large effect in the placebo plus antidepressant arm (15.8 points), possibly due to the hours of human contact involved. It is also less than the 6.5 point difference Janssen used in their sample size calculation (p. 91 and p. 157 in Ref. 2). More importantly, the time point of 4 weeks in all these studies means the data are rather uninformative, since treatment-resistant depression is usually treated for months or years.

\section{Food and Drug Administration (FDA)}

Kasper et al consider that the regulatory agencies have employed 'careful consideration'. The FDA's convention to request two shortterm studies to approve the efficacy of a drug ('each convincing on its own') has been criticised because it allows companies to conduct as many studies as are necessary to generate two positive studies. However, even that low bar was dropped: in 2014, in discussion with Janssen, the FDA 'agreed' that a withdrawal study could be used as one of two positive studies, 'along with a short-term fixed-dose study with statistically very persuasive results' (italics ours, p. 27 of Ref. 2). However, after further meetings with Janssen this was 'later switched to any short-term study in March 2018' (italics ours, p. 27 of Ref. 2). Many other commentators and national health service bodies, including the National Institute for Health and Care Excellence (NICE), have drawn different conclusions from those of the FDA and the Medicines and Healthcare Products Regulatory Agency and questioned the data on the safety and efficacy of esketamine. ${ }^{3}$

\section{Suicide}

We acknowledge that comparing data from non-randomised groups (as in Table 1 in the Analysis) cannot establish causal attribution and that the larger numbers in the esketamine group and the longer duration of treatment might have inflated suicides in this group; also that participants might have a relatively high baseline rate of suicide. However, the meta-analysis identified by Kasper is not an appropriate comparison. The Janssen studies included people who had only 'failed' two antidepressants (which according to the STAR-D trial probably represents at least $44 \%$ of patients with depression) and excluded people with a recent history of suicidal intention, psychiatric co-morbidity, drug and alcohol problems, vagal nerve stimulation (VNS) and deep brain stimulation, ${ }^{2}$ whereas the meta-analysis involved a more severe group of patients
Table 1 Withdrawal symptoms recorded 4 weeks after stopping esketamine in the safety trial (adapted from Supplementary Table 5 ) to two significant figures

New or worsened symptom at week 4 Proportion (\%)

Loss of appetite

Nausea/vomiting

Diarrhoea $14 \%$

Diarrhoea $1.8 \%$ $7.1 \%$

Irritability

Dysphoric mood/depression $\quad 23 \%$

Insomnia

Fatigue/lethargy/lack of energy

Poor coordination

Restlessness/agitation

Diaphoresis

Tremor/tremulousness

Dizziness/light headedness

Headaches

Muscle aches and stiffness

Weakness

increased acuity to sound, smell or touch

Paraesthesias

Difficulty concentrating, remembering

Depersonalisation/derealisation

$27 \%$

$16 \%$

$5.4 \%$

$5.4 \%$

$8.9 \%$

$7.1 \%$

$8.9 \%$

$11 \%$

$8.9 \%$

$5.4 \%$

$3.6 \%$

$5.4 \%$

$18 \%$

$1.8 \%$

trialling ECT, deep brain stimulation and VNS among other 'end of the line' treatments. Furthermore, in the safety study, one in seven patients developed 'treatment-emergent' suicidal ideations, and six attempted suicide in a group selected for not being actively suicidal; ${ }^{4}$ a disproportionate number of suicides have been attributed to esketamine in the first year of its use in the USA. ${ }^{5}$

\section{Adverse effects}

Even with weekly or fortnightly dosing, $17 \%$ of patients (136/802) in the long-term safety study demonstrated symptoms reminiscent of 'ketamine bladder', a known and potentially serious complication of ketamine use. ${ }^{4}$ Jauhar et al and Kasper et al reiterate the FDA's claim that most of the bladder-related side-effects were transient and mild, but even in the shorter trials 33\% of cases were not minor, and $24 \%$ of cases had not resolved at the subject's last assessment (p. 46 of Ref. 2). The FDA also commented that serious bladder conditions may have been missed or misidentified (p. 46 of Ref. 2).

\section{Withdrawal and relapse}

As recognised by Kasper et al, ketamine causes tolerance, dependence and withdrawal, and the doses of esketamine employed in the studies were similar to recreational doses of ketamine. As Jauhar et al report, the FDA and Janssen claimed that withdrawal symptoms were probably not relevant in the relapse prevention study, but Janssen did not report the Physician Withdrawal Checklist data to justify this conclusion. However, Janssen did describe withdrawal effects ('new or worsened' effects) in the longer safety study shown in Table $1,{ }^{4}$ all recognised ketamine withdrawal effects. The presence of symptoms such as paraesthesia, diarrhoea and diaphoresis, occurring in concert with psychological symptoms, marks this as distinct from relapse.

Although it is difficult to be definitive about the nature of experiences that occur following drug discontinuation, the possibility that withdrawal effects were mistaken for relapse requires consideration, as withdrawal effects overlap with most items on the Montgomery-Åsberg Depression Rating Scale. NICE concluded 
that 'any withdrawal effect would be difficult to distinguish from a change in depressive symptoms' (pp. 14-15 of Ref. 3).

\section{High}

Ketamine, like some other anaesthetics, causes a pleasurable 'high' in some users and reduces depression scores within hours, and it is not clear how drug-induced euphoria and antidepressant effects can be distinguished. Jauhar argues it is the persistence of the effect that marks it as 'antidepressant', but, as described above, the esketamine trials do not confirm that a clinically relevant effect occurs.

\section{Language}

We apologise if the term 'guinea pigs', referring to potential trial participants, caused offence and perhaps could have chosen our words differently. We used this strong language to communicate our concern that the public might be exposed to a pharmacological agent for which robust efficacy and safety have not yet been demonstrated.

\section{Conclusion}

Overall, the central points of our Analysis remain: esketamine has a clinically uncertain effect at 4 weeks, and there are no studies with longer follow-up periods more relevant for the care of people with depression. The discontinuation trial potentially conflates relapse and withdrawal and there are concerning safety signals.

\section{Funding}

None.

\section{References}

1 Leucht S, Fennema H, Engel RR, Kaspers-Janssen M, Lepping P, Szeged A. What does the MADRS mean? Equipercentile linking with the CGI using a company database of mirtazapine studies. J Affect Disord 2017; 210: 287-93.

2 Food and Drug Administration. Efficacy, Safety, and Risk-Benefit Profile of New Drug Application (NDA) 211243, Esketamine 28 mg Single-Use Nasal Spray Device, submitted by Janssen Pharmaceuticals, Inc., for the Treatment of Treatment-Resistant Depression. FDA, 2019. Available from: https://www.fda. gov/media/121376/download.

3 National Institute for Health and Care Excellence. Esketamine for Treating Treatment-Resistant Depression: Appraisal Consultation Document. NICE, 2020

4 Wajs E, Aluisio L, Holder R, Daly EJ, Lane R, Lim P, et al. Esketamine nasal spray plus oral antidepressant in patients with treatment-resistant depression: assessment of long-term safety in a phase 3, open-label study (SUSTAIN-2). $J$ Clin Psychiatry 2020; 81(3): 19m12891.

5 Gastaldon C, Raschi E, Kane JM, Barbui C, Schoretsanitis G. Post-marketing safety concerns with esketamine: a disproportionality analysis of spontaneous reports submitted to the FDA adverse event reporting system. Psychother Psychosom 2021; 90(1): 41-8.

Mark Horowitz, Clinical Research Fellow, University College London, London, UK. Email: mark_horo@hotmail.com; Joanna Moncrieff, Professor of Critical and Social

Psychiatry, University College London, London, UK

doi:10.1192/bjp.2021.163 